\title{
M-ATCP: Modified-ATCP for Mobile Ad-Hoc Networks
}

\author{
Alhamali Masoud Alfrgani Ali \\ Department of Computer Science \&IT \\ SHIATS, (Deemed University) Naini \\ Allahabad- India
}

\author{
Raghav Yadav \\ Department of Computer science \& IT \\ SHIATS, (Deemed University) Naini \\ Allahabad- India
}

\begin{abstract}
In TCP/IP suite transport layer protocol Transmission Control Protocol (TCP) was initially used for secure connectionoriented host-to-host transmission of the packet over wired networks. When this TCP is implement over wireless mobile networks environments, its leads to reduced throughput. It has been observed that when the wired TCP is apply in wireless environments, problems are not always due to network congestion state, but rather they are mostly because of high bit error rates, frequency of route failure, and topological network changes. Researchers have suggested a solution to inset a layer employment, a new layer between network and transport layer called ATCP (ad-hoc TCP). ATCP works over the sender node only and assuming that the sender node being always reachable: which is not always possible to meet in wireless mobile networks environments. Authors proposed an approach based on the local route request (LRREQ) packet, which is capable of mitigating ATCP, the assumption is that the sender node is always in the reachable state as well as intermediate link failure, which is also repaired with LRREQ mechanism.
\end{abstract}

\section{Keywords}

TCP; Wireless Networks; Congestion Control; Mobility; Mobile Ad-hoc.

\section{INTRODUCTION}

Transmission Control Protocol (TCP) was initially used for connection-oriented for source-to-destination delivery of packet in wired networks. When packet losses appear i.e. throughput degradation of the network, it indicates congestion state in the network. Transport layer protocol (TCP) has not any information whether the Internet Protocol (IP) is running over wired or wireless environments. But in the case of wireless networks, don't have a static system like wired networks. These types of networks are mostly deployed for temporary networks connectivity such as disaster relief or on the battlefield etc. In Ad-hoc network, each mobile node acts as a router and destination node, which transfer and forward the packets from source to destination node. TCP is too much suitable for wired network, whereas for wireless same TCP is not efficient like wired networks: due to node mobility and sharing of wireless communication links. Overlooking of wireless properties can be lead to TCP implementations with very poor performance; behind this the cause of TCP lies in performing congestion control in case of losses which are not induced by network congestion state. Actually, bit-error rate (BER) is very high in wireless as compared to wired networks and frequently topological changes in wireless networks are also very high. Due to high BER error, data packets are corrupted, and as result acknowledgments (ACK) issued by TCP are considered to be lost. If sender node doesn't receive the ACK within the specified time frame i.e. retransmission timeout (RTO), then the sender node assumes that somewhere in the route there is, congestion. Sender exponentially reduces its congestion control window, this type of situations are repeatedly monitored by sender node, resulting in the very low throughput of the networks. So many authors research worked had been proposed to make more efficient TCP performance over MANET [1] [2] [3] .The effect of topological changes i.e. re-route finding after the failure of existing route, detected by sender node, then network layer of sender node try to a setup anew route to destination node. The probability is very high, that before a new route is establish the RTO will be expired. As a result sender node retransmits lost packet at the same time, which also invokes congestion control window. When new route is successfully setup, and packets delivered through the new route, sender TCP slowly starts its congestion control window. If the mobility of nodes is very high in case of MANET, then it tends to frequent recomputation of routes, thus reduces network throughput drastically. Based on these problems, researchers were bound to think to improve TCP performance over MANET; the proposed solutions are: introduced a new layer that layered suggestions are comparatively more efficient. In new layer proposal, transport layer (TCP) and network layer (IP) protocols work jointly, then significant improvident are possible where TCP is capable to make differentiate that the packet losses due to congestion or losses due to topological changes in network; two properties discussed i.e. high biterror rate and frequently re-route finding after failure detected in route. This new layer approach is motivated to if lower layer's information transfer to upper layer which helps to improve upper layer performance better and easy manner. To achieving this, when the layer detects en-route failure, it sends notice to the TCP sender node about the failure change in route [4], [5], [6], [7], [8]. Once receiving this notification, TCP sender node will be enter in freezing state. Then TCP stops sending data packets, and it freezes all parameter with value at this time, like: congestion windows size and transmission timer. After new route re-establishment than TCP sender node enter to normal state. The cross-layer methods are more suitable for short-term performance optimization of networks, i.e. high mobility of nodes. In the layered methods, the problems of TCP are introduced at one new thin OSI layers. In this approach, adaptive TCP delayed acknowledgements is used to minimize the conflict on wireless links. But other hands layered methods applicable only on the concept of designing of protocols in separation, thus long term solution can be achieved by layered methods. We find that, cross-layer approach reported good performance than layered methods.

Cross-layered approach does not try to moderate working of standard TCP, because that the important issues is how to maintain compatibility with TCP/IP suite, in this condition only thin layer is introduced between transport and network layer protocol in standard version of OSI reference model called as an ATCP (ad-hoc TCP) layer. ATCP works on the network condition information, which is provided by explicit congestion notification (ECN) packet [1], [3], and another protocol ICMP "Destination Unreachable" messages, then 
TCP at the sender node put to the appropriate state. At the same time other hand, on receipt of a "Destination Unreachable" packet, then TCP state enter at the sender node is frozen state until a new route is established and make sure that the sender node does not initiate congestion control window. Furthermore, the sender node not send's any packet into the network, during the period when no route exists between the source and destination node. The ECN packet is used to explicitly notify to sender node about the congestion state of the network and route is currently in use. When it is received then normally TCP congestion control window invoked without waiting timeout of RTO. For the detection of losses packets due to channel error is based on ACK's count. When ATCP received continuously three duplicate ACK's, it does not issue third duplicate Acknowledgement but at the same time to puts TCP in the buffer state and very efficient manner retransmits the lost packet from TCP's buffer. When ATCP received the next Acknowledgement then it will be try to resume normal working of TCP. The important issue of ATCP is to allow interoperability with TCP sources or destinations that do not implement ATCP. At the same time ATCP does not affected the normal operation of TCP's functioning in cases of hybrid type of environment communication links such as: the TCP connection is between a node in the wire-line network and wireless network. ATCP can minimize the problem of high BER, network congestion, and positioning of the packets. At the same time it is very difficult or impossible to assume that ECN capable nodes and sender node is always in reachable state somehow hard to meet in a mobile Ad-hoc system. In this paper the author has considered two problems - the first being that, while implementing ATCP, the researchers had always assumed that the sender node is always reachable, though it is not always true and the second being, the re-route establishment. The remainder of this paper is organized as follows. Section II\& III discuss literature review and proposed approach. Section IV, simulation parameters \&results and section $\mathrm{V}$ final conclusion.

\section{LITERATURE REVIEW}

A number of approaches had been put forth by many authors for the enhancement in the presentation of the TCP over wireless networks [4] [5] [9] [10], as the performance deteriorates, when the TCP, which was designed for wired networks, is used in the wireless environment. In wireless networks, the TCP is the working connection for multiple wireless channels. According to [4] A. Al Hanbali et al. in this research, the authors have presented a vital role of mobility issue and also present elaborative discussion of the vital factors that are associated with the wireless network. Overhere the authors have also explained, that how the TCP can be influenced by the mobility issue and the lower layer's protocols. [11]D. Kim, et al. suggests a new approach for the improvement of TCP's performance in wireless network which has been substantiated by the results, where in each node has a capable to buffering of packets, in transferring and during a route failure state and route re-establishment state, while simultaneously the authors have a view that the differences between the network congestion state and the route disconnection state are due to the topological changes caused by the node mobility, they also recommended changes for the secure transmission and manipulation for the control packets of TCP's fast and efficient recovery scheme[12].

$X$. Zhang, et al. [8] suggested a new method which is based on compression between, MAC channel efficiency and virtual TCP performance, also compare to virtual TCP's channel efficiency. The authors had also tabulated that the performance of the suggested method, out performs the 802.11 DCF, in terms of vital parameters of network such as throughput and delay. The authors have also, quantified the real channel efficiency in MAC protocol with the virtual channel efficiency in TCP, it can also control the data transmission rate of TCP by using the congestion control window and the flow of TCP acknowledgements, to enhance the data transmission rate of MAC protocol before feeling of congestion in network and to enhance the performance of the network. [13] The researchers introduces the Congestion Free Routing in Ad-hoc Networks (CFR), which is based on the dynamic estimation of the network congestion state by calculating the average queue length at the node level in the network. [14] The foundation of this study is the mobile agent based congestion control approach. In this suggested approach is more suitable to avoid congestion in the Ad-hoc network, with the help of mobile agent traverses throughout the network and calculates a less-loaded neighbor node as its next hop for the route. The routing table is also updated as per the collected data of each node, of congestion status. The dynamic network topology changes can be updated in the routing table with the information gathered by the mobile agents, the nodes can be updated at the same time. [15]. this study is based on the fact, that the congestion of the wireless local area networks is affected by the performance of the transport layer protocol. The authors have presented an analysis based solution which is known as Crosslayer congestion avoidance scheme (C3TCP) and have discussed its implementation view in detail, which is also assistive in obtaining enhanced performance by collecting capacity information such as bandwidth and delay at the data link layer. The method needs, the introduction of an additional module within the protocol stack of the mobile node, which can adjust the outgoing data stream based on capacity measurements. The authors have also suggested a proposal to provide an optional field support to the existing IEEE 802.11 protocol, to support the presented congestion control solution as well as many other similar approaches. The outcome of this analysis shows, the high utilization of all the available resources in the designing phase of the protocol.

\section{PROPOSED APPROACH}

\section{Local Route Request (LRREQ) based Route Repairing}

The wireless node networks are well established, but the major problem arises in these types of network's link failure. Then it is compulsory to repair failed route as soon as possible, this can be achieved by two types of repairing methods (a) when source node not reachable (Modification in ATCP) and (b) local repairing in route

\subsection{When source node not reachable (Modification in ATCP)}

In fig.1, a mobile topology has been depicted, where assume that a path from source node $\mathrm{N} 3$ to destination node N12 is setup, the path is $\mathrm{N} 3 \rightarrow \mathrm{N} 6 \rightarrow \mathrm{N} 7 \rightarrow \mathrm{N} 10 \rightarrow \mathrm{N} 12$. Now after sometime, due to the mobility of the node, it might happen that the source node $\mathrm{N} 3$ moves out of coverage range of the neighboring node N6, which causes a link failure between N3 and N6 nodes.

According to proposed approach i.e. LRREQ, a partial path between $\mathrm{N} 3$ and $\mathrm{N} 6$ will be setup; the remaining path i.e. $\mathrm{N} 6 \rightarrow \mathrm{N} 7 \rightarrow \mathrm{N} 10 \rightarrow \mathrm{N} 12$ will remain the same. Now, as per the proposed approach i.e. LRREQ, the new path of source (N3) and destination (N12) node will be setup as: $\mathrm{N} 3 \rightarrow \mathrm{N} 1 \rightarrow \mathrm{N} 6 \rightarrow \mathrm{N} 7 \rightarrow \mathrm{N} 10 \rightarrow \mathrm{N} 12$. 


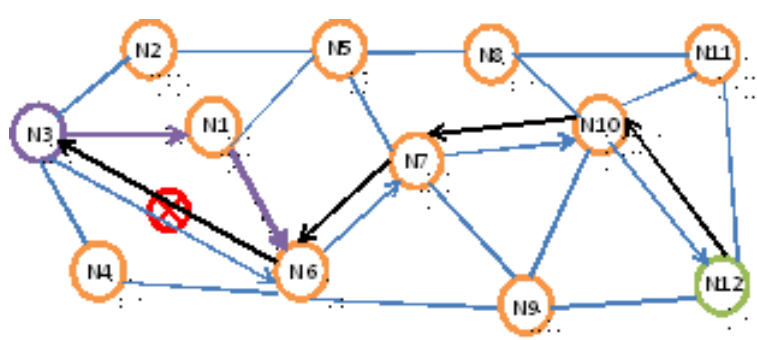

Figure 1: Source Node Not Reachable (Modification In Atcp)

\subsection{Local Repairing in Route (LRREQ)}

In this case it is the responsibility of node (N10) to broadcast local route request (LRREQ) packets to setup a new partial path between node (N10) and (N12). For example in this topology a partial route will be setup with the help of node (N11), then the resulting path will be $\mathrm{N} 3 \rightarrow \mathrm{N} 6 \rightarrow \mathrm{N} 7 \rightarrow \mathrm{N} 10 \rightarrow \mathrm{N} 11 \rightarrow \mathrm{N} 12$.

Shown fig. 2 in case (a) the value of TTL will be increased, it is permitted that TTL $<=2$, that means in this case network can take two hop counts to bypass failure network. It is permitted in a consecutive order; first node N10 will discover the one node to bypass the network if it not possible then it will go for the second nodes to establish the network. If such case arises when required more than two nodes at this time node N10 will send the RERR to source node and source node will discover the new route to transmit the data.

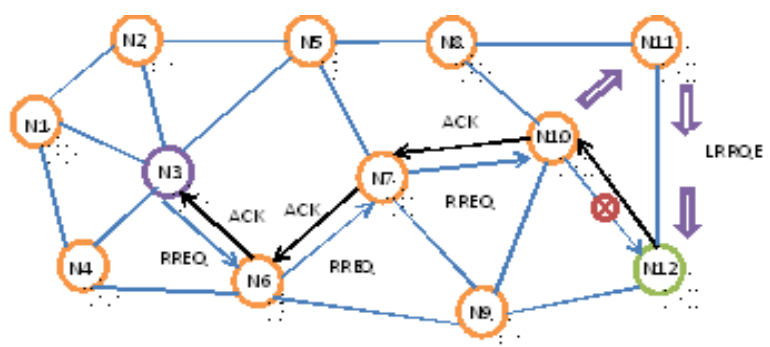

Figure 2: Local Repairing In Route

Local Repair flowchart

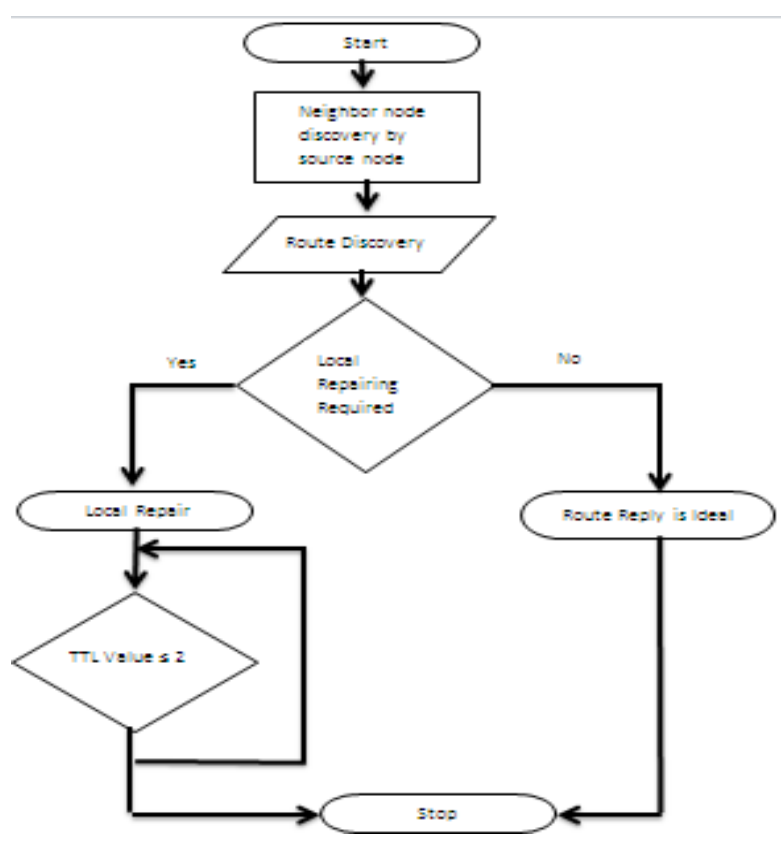

Figure 3: Algorithm Of The Lrreq
In this proposed approach, when upstream nodes find a link failure, then it is responsibility of upstream node to sends an LRREQ message, only towards the destination. This procedure contributes towards reducing the hop count as defined in $\mathrm{TTL}<=2$.In fig 3 shows a flow chart of the proposed local route repair procedure.

\section{Matlab Simulation Algorithm}

Used Terms:

Send Data: SD

Data Received: DR

Acknowledgement Received: ACKR

Retransmission Time Out: RTO

Disconnection: Disc

Connection: Conn

Network Status: NS

Function Initialize()

If data is sent in memory buffer and sending window is open

Return sending;

Else if sending window is close and wait for update of acknowledgement

Return waiting;

Else

Return done

End

Function SD ()

If $\mathrm{NS}==$ Conn

Normal TCP Working;

Else

Memory buffers the Data;

End

\section{Function Disc ()}

If $\mathrm{NS}==\mathrm{Disc}$

Initialize=Initialize () ;

If initialize $=$ done

No action;

Else if initialize $==$ sending

No retransmission;

Note sequence number of last packet sent;

Else if initialize $==$ waiting

RTO=FALSE;

Disc $=$ TRUE;

End

\section{Function Conn ()}

NS==Conn;

If initialize $=$ done 
No action;

Else if initialize $==$ sending

Resume sending data from sent packet;

Else if initialize==waiting

If $\mathrm{RTO}==\mathrm{TRUE}$

Retransmit last sent packet;

RTO=FALSE

Else

No action;

End

End

Function RTO ()

If $\mathrm{NS}==\mathrm{Conn}$

Done;

Else

RTO=TRUE;

End

\section{SIMULATION PARAMETERS AND RESULTS}

Table 1: Parameter For Simulation

\begin{tabular}{|l|l|}
\hline Time & $20 \mathrm{Sec}$. \\
\hline Area & $12 \mathrm{~km} \times 12 \mathrm{~km}$ \\
\hline Coverage Area & $320 \mathrm{~m}$ \\
\hline Mobility of Node & $8 \mathrm{M} / \mathrm{Sec}, 24 \mathrm{M} / \mathrm{Sec}$ \\
\hline Packet Size & 512 Bytes \\
\hline No. of Nodes & $25 \sim 100$ \\
\hline Data Transfer Rate & $4.2 \mathrm{kBps}$ \\
\hline Field Name & Description \\
\hline Sid & Source Node ID \\
\hline Did & Destination Node ID \\
\hline Ids & Sender Node ID \\
\hline Hc & Hop count of Sender Node \\
\hline
\end{tabular}

\section{Throughput Analysis}

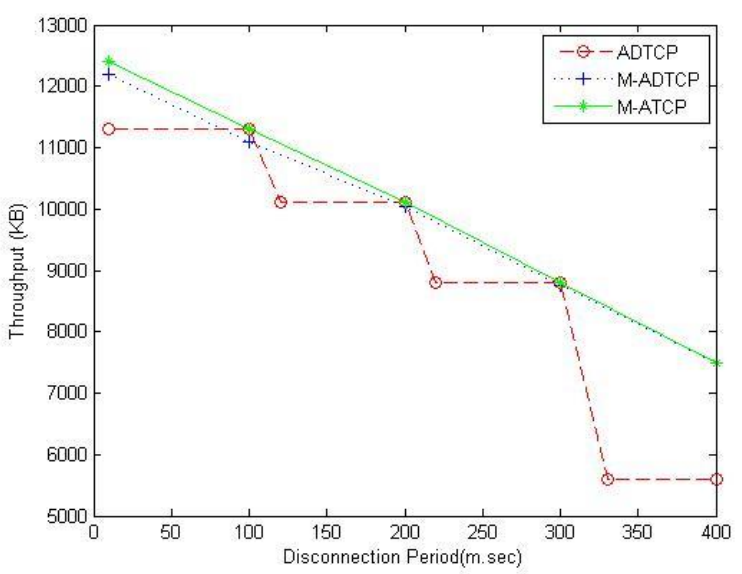

Figure 4: Throughput Analysis

Observations resulting from the simulation are as follows:

M-ATCP shows more enhancement in throughput as compared to ADTCP and M -ADTCP, as the disconnection interval increases. This increase in throughput for M-ATCP is due to the local repairing of the route by sending LRREQ to in route nodes. In fig. $4 \mathrm{M}$-ATCP gives the maximum throughput than other disconnection periods.

It is also observed that the performance of M-ATCP is very close to that of M-ADTCP, in small disconnection period. MATCP showed an improvement of up to $20 \%$ over ADTCP for long disconnection intervals as shown in fig. 4 the idle period after reconnection is the primary factor in degrading the throughput rather than the reduction in the congestion window. Shown in fig.4,proposed M-ATCP performs better than both ADTCP and M-ADTCP schemes.

\section{Congestion window Analysis}

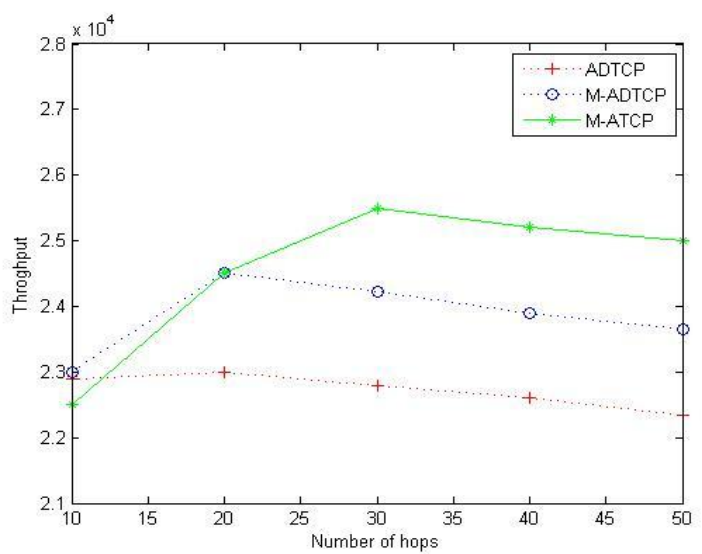

Figure 5: Throughput Comparisons

In fig. 5 shows different throughput of ADTCP, M-ADTCP and M-ATCP. The throughput of ADTCP protocol is very much lower than others two protocols. When data transfer rate is high i.e. traffic increases, then relatively throughput of ADTCP not increases, although the throughput of another ADTCP and M-ADTCP protocols increases significantly. This is concluded that ADTCP does not utilize networks resource effectively manner. The data transmission rate increases more, then M-ADTCP does not support improvements in the throughput effectively. It is happened because it does not calculated the available bandwidth and 
also, blocks the buffer repeatedly, it will also delay the sender receiving ACK process. At the under high data traffic load condition, M-ATCP system is still reaching at high throughput.

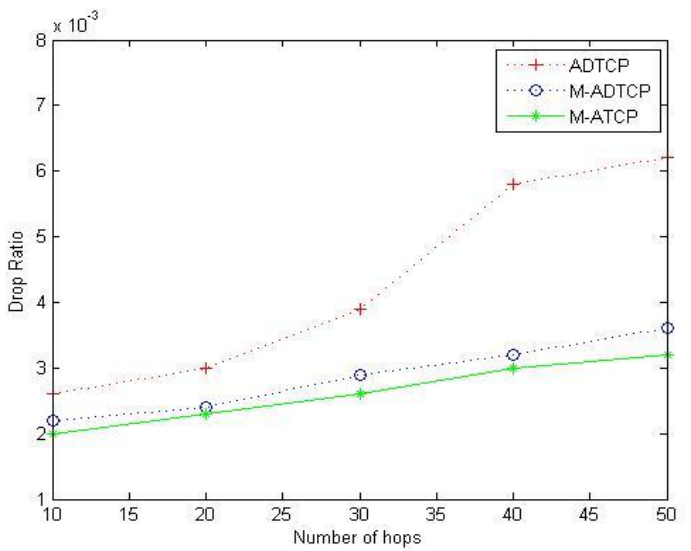

Figure 6: Drop Ratio Comparisons

In fig.6 is displaying the drop ratio of comparative study of the different protocols. From analysis that shows the changes in graph is due to small size in queue length, M-ATCP have lower drop ratio. Where other two protocols ADTCP and MATDTCP are showing higher drop ratio than M-ATCP. It also shows $10 \%-15 \%$ improvement by M-ATCP protocol.

\section{CONCLUSION}

Authors proposed a modified ATCP (M-ATCP) for wireless mobile network. In such network local route request (LRREQ) will work when source node or any in-route node is unable to forward the data packets to its neighbor node. It is found that in mobile network, the disconnection period and drop ratio will be reduced by using LRREQ. M- ATCP works more effectively than other two methods ADTCP and MADCP. M-ATCP has solved the problem of new route setup when source node will not be reachable during the transmission of data. It is also working effectively when an inroute node is unable to send the data packets to the destination node. Thus, we have concluded that M-ATCP worked effectively than other methods in wireless mobile network topology.

\section{REFERENCES}

[1] Jian Liu, and Suresh Singh, "ATCP: TCP for mobile ad hoc networks", IEEE Journal.

[2] A. Al Hanbali, E. Altman, And P. Nain, Inria Sophia Antipolis France, "A survey of TCP over ad-hoc networks", IEEE Communications surveys \& tutorials, vol.7, issue: 3 , pp. 22 - 36, 2005
[3] Ajay Kumar Singh, and Sridhar Iyer, "ATCP: Improving TCP performance over mobile wireless environments", In Proceedings of $4^{\text {th }}$ International workshop on mobile and wireless communications network, pp. 239-243, 2002.

[4] K. Chandran, S. Raghunathan, S. Venkatesan, and R. Prakash, "A Feedback based scheme for improving TCP performance in ad hoc wireless networks," in the Conference on Distributed Computing Systems, Amsterdam, Netherlands, May 1998, pp. 472-479.

[5] G. Holland and N. Vaidya, "Analysis of TCP performance over mobile ad hoc networks," $A C M$ Wireless Networks, vol. 8, no. 2, pp. 275-288, Mar. 2002.

[6] J. Liu and S. Singh, "ATCP: TCP for mobile ad hoc networks," IEEE JSAC, vol. 19, no. 7, pp. 1300-1315, Jul. 2001.

[7] F. Wang and Y. Zhang, "Improving TCP performance over mobile ad hoc networks with out-of-order detection and response," in Proc.of ACM MOBIHOC, Lausanne, Switzerland, Jun. 2002, pp. 217-225.

[8] D. Kim, C. Toh, and Y. Choi, "TCP-BuS: Improving TCP performance in wireless ad hoc networks," Journal of Communications and Networks, vol. 3, no. 2, pp. 175186, Jun. 2001.

[9] M. Frodigh, P. Johansson, and P. Larsson, "Wireless adhoc networking:the art of networking without a network", Ericsson review, vol. no.4, pp. 248-263, 2000.

[10] C. Lochert, B. Scheuermann, and M. Mauve, "A survey on congestion control for mobile ad-hoc networks", Wireless communications and mobile computing, vol. 7, issue 5, pp. 655-676, 2007.

[11] D. Kim, C.-K. Toh, and Y. Choi., "TCP-Bus: Improving TCP performance in wireless ad-hoc networks", In Proceedings of the IEEE International conference on communications, vol.3, pp. 1707-1713, 2000.

[12] Xinming Zhang, Jun Lv, Xiaojun Han, and Dan Keun Sung, "Channel efficiency-based transmission rate control for congestion avoidance in wireless ad-hoc networks", IEEE communications letters, vol. 13, no. 9, 2009

[13] T. S Kumaran, and V. Sankaranarayanan, " Congestion free routing in ad-hoc networks", Journal of computer science, vol. 8, no 6, pp. 971-977, 2012.

[14] D. Kliazovich, and F. Granelli, "Cross-layer congestion control in ad-hoc wireless networks", Ad-hoc networks, vol. 4, issue 6, pp. 687-708, 2006.

[15] A K Mourya, and N Singhal, "Managing congestion control in mobile ad-hoc network using mobile agents", International journal of computer engineering \& applications, vol. IV, Issue I/III, 2013.

[16] SachinGajjar, and Hari M. Gupta, "Improving performance of ad-hoc TCP in mobile ad-hoc networks"India Conference, 2008. INDICON 2008, pp. 144-147. 